\title{
Binary hard sphere mixtures in block copolymer melts
}

\author{
Russell B. Thompson, ${ }^{1}$ Jae Youn Lee, ${ }^{1}$ David Jasnow, ${ }^{2}$ and Anna C. Balazs ${ }^{1}$ \\ ${ }^{1}$ Department of Chemical and Petroleum Engineering, University of Pittsburgh, Pittsburgh, Pennsylvania 15261 \\ ${ }^{2}$ Department of Physics and Astronomy, University of Pittsburgh, Pittsburgh, Pennsylvania 15260
}

(Received 12 February 2002; published 17 September 2002)

\begin{abstract}
We perform a self-consistent-field/density-functional-theory hybrid analysis for a system of diblock copolymers mixed with polydisperse, hard, spherical particles of various chemical species. We apply this theory to study the equilibrium morphologies of two different binary sphere/diblock melts. First, we examine the case where the particles have two different sizes, but both types are preferentially wetted by one of the copolymer blocks. We find that the single-particle distributions for the two species do not track one another and that the particles show a degree of entropically generated separation based on size, due to confinement within the diblock matrix. Second, we study the case where the particles are all the same size, but are of two different chemical species. We find that, as expected, the particle distributions reveal a degree of enthalpically driven separation, due to the spheres' preferential affinities for different blocks of the copolymer.
\end{abstract}

DOI: 10.1103/PhysRevE.66.031801

PACS number(s): 83.80.Uv, 82.70.-y

\section{INTRODUCTION}

The combination of organic polymers and inorganic particles can lead to a composite material that is more useful than either of the individual components [1]. By careful selection of the particles and polymers, designers can tailor composites to meet the final product requirements that might be difficult to achieve using other materials. Currently, there is tremendous interest in forming organic/inorganic composites that are spatially organized on the nanometer length scale. Such nanostructured composites are needed to overcome obstacles to miniaturization as feature sizes in devices reach the nanoscale.

The most efficient route for creating these composites is through self-assembly, where cooperative effects between the different components drive the system to form nanostructured materials [2]. Block copolymer/particle mixtures constitute ideal self-assembling systems for creating nanostructured materials since the microphase separation of block copolymers can be harnessed to template the ordering of the particles and yield hybrid materials that are ordered on the nanoscale. Recent experimental [3-5] and theoretical [6-9] studies have, in fact, shown that when nanoparticles are preferentially wetted by one of the blocks of a diblock copolymer, the system can form spatially periodic nanocomposites.

In our previous theoretical studies of nanoparticle/diblock mixtures [6-9], the particles were taken to be monodisperse in size. In this paper, we extend the hybrid self-consistentfield theory/density-functional-theory (SCFT/DFT) methodology to deal with multiple species of particles added to the copolymer matrix. We show that there is a rich phase behavior that emerges when we introduce particles that differ in size into the microphase separating $A B$ diblocks. Specifically, we first consider the case where the particles are bidisperse in size and chemically identical; that is, both the smaller and larger particles are preferentially wetted by the $A$ block. Binary mixtures of particles that differ in size can undergo entropically driven phase separation and order into a variety of crystal structures [10-13]. This organization normally occurs at relatively high volume fractions of particles and at relatively large disparity in particle sizes. As we see below, in the presence of the diblocks, a low global volume fraction of bidisperse particles undergoes a unique self-assembly within the $A$ domains. The particles organize into "graded films," where the large particles are localized in the center of the domains while the smaller particles tend to be located at the edges of these regions. This type of organization is sufficiently general that it is found in the lamellar, cylindrical, and spherical phases of the system. If the particles are semiconductors, the graded layers could display novel optoelectronic properties [14].

In the second application of the SCFT/DFT approach, we consider the case where the particles are identical in size but differ in chemical nature; that is, one type of particle is preferentially wetted by the $A$ units, while the other particles are wetted by the $B$ species. In the presence of the $A B$ diblocks, the particles clearly are enthalpically driven into the respective compatible domains and thus can form other types of ordered organic/inorganic hybrid materials.

Common to both these cases is the fact that the selforganizing diblock matrix promotes the self-assembly of the particles: at one extreme the organization is entropically driven, while at the other extreme, it is enthalpically driven. Ultimately, the competition of enthalpic and entropic effects may be further exploited, for example, combining different affinities with a bidisperse or other distribution of particle sizes. The systematic probing of the structures one may achieve is beyond the scope of the present work. These examples illustrate that self-assembly in systems composed of binary particle mixtures and block copolymers can be exploited to create novel, spatially organized nanocomposites.

In the following section, we discuss our extension of the hybrid SCFT/DFT theory that describes mixtures of diblock copolymer melts and polydisperse, hard, spherical particles. For block copolymer systems, SCFT has yielded quantitative agreement with experimental studies $[15,16]$, and it remains the method of choice for numerical studies of potential equilibrium phases. DFT is similarly an appropriate choice for studying inhomogeneous fluids of various colloidal particles [17]. Our approach here represents an extension of previous 
methodologies and calculations $[7,8]$, in which the combined SCFT/DFT theory allowed the isolation of new selfassembled morphologies where diblocks and monodisperse particles spontaneously order into a mesoscopically regular pattern.

In Sec. III, we first present the results for the case where the particles are of two different sizes, but are chemically identical and have a preferential interaction with one of the copolymer blocks. We follow this with a description of the situation where the particles are monodisperse in size but half of the particles have an affinity for the $A$ block, while the other half have an affinity for the $B$ block of the diblocks. We summarize our conclusions in the last section.

\section{THEORY}

Consider a system of $n_{d} A B$ diblock copolymers together with hard spherical particles of various radii. Let us divide the particles into $m$ groups based on their radii and chemical species such that $n_{p 1}$ is the number of particles of chemical type $p_{1}$ and radius $R_{1}, n_{p 2}$ is the number of type $p_{2}$ and radius $R_{2}$, or in general, $n_{p i}$ is the number of type $p_{i}$ and radius $R_{i}$. Each copolymer $\alpha$ is parametrized along a space curve $\mathbf{r}_{\alpha}(s)$ with a variable $s$ that runs from 0 to 1 . The partition function for this system is

$$
\begin{aligned}
& Z=\frac{1}{n_{d} ! \prod_{i}\left(n_{p i} !\right)} \int \prod_{\alpha=1}^{n_{d}} \widetilde{\mathcal{D}} \mathbf{r}_{\alpha}\left[\prod_{i}\left(\prod_{\beta_{i}=1}^{n_{p i}} d \mathbf{R}_{\beta_{i}}\right)\right] \\
& \times \delta\left[1-\sum_{\mu} \hat{\varphi}_{\mu}(\mathbf{r})\right] \\
& \times \exp \left[-\frac{\rho_{0}}{2} \int d \mathbf{r} \sum_{\substack{\mu, \nu \\
\mu \neq \nu}} \chi_{\mu \nu} \hat{\varphi}_{\mu}(\mathbf{r}) \hat{\varphi}_{\nu}(\mathbf{r})\right] .
\end{aligned}
$$

In this work, we shall let Greek indices run over all the various chemical species, $A, B, p_{1}, p_{2}, \ldots, p_{m}$, while Latin indices run over only the $m$ particle species, unless the products or summations are explicitly labeled otherwise. Therefore, $\chi_{\mu \nu}$ represents the Flory-Huggins parameters between the different constituents of the mixture. The segment volume $\rho_{0}^{-1}$ is taken to be identical for all species. For the case where there is only one type of particle $p_{1}$, expression (1) reduces to the partition function for a diblock/monodisperse hard sphere composite [8]. The $\delta$ function enforces incompressibility and $\widetilde{\mathcal{D}} \mathbf{r}$ is a Gaussian weighted functional integral [8]. $\mathbf{R}_{\beta_{i}}$ is the position of the center of the $\beta$ th particle of species $i$. The form of the concentration operators $\left\{\hat{\varphi}_{\mu}(\mathbf{r})\right\}$ has been given previously [8], and following the derivation in that work we find the mean field free energy for our current system to be [18]

$$
\begin{aligned}
\frac{N F}{\rho_{0} k_{B} T V}= & -\sum_{i} \frac{\phi_{i}}{\alpha_{i}} \ln \left(\frac{Q_{i} \alpha_{i}}{V \phi_{i}}\right) \\
& -\left(1-\sum_{i} \phi_{i}\right) \ln \left[\frac{Q_{d}}{V\left(1-\sum_{j} \phi_{j}\right)}\right] \\
& +\frac{1}{V} \int d \mathbf{r}\left[\frac{1}{2} \sum_{\mu, \nu} \chi_{\mu \nu} N \varphi_{\mu} \varphi_{\nu}-\sum_{k=A, B} w_{k} \varphi_{k}\right. \\
& \left.-\sum_{i} w_{i} \rho_{i}-\xi\left(1-\sum_{\mu} \varphi_{\mu}\right)\right]
\end{aligned}
$$

Here, $\Sigma_{k=A, B}$ denotes a summation over only the copolymer species $A$ and $B$. The functions $\left\{\varphi_{\mu}(\mathbf{r})\right\}$ are now interpreted as the ensemble averages of the local volume fractions, while $\left\{\phi_{\mu}\right\}$ are the constant overall volume fractions of the various constituents. Also, $\left\{\rho_{i}(\mathbf{r})\right\}$ are the ensemble averages of the particle center distributions. The mean fields $\left\{w_{\mu}(\mathbf{r})\right\}$ have been introduced to account for the interactions in the system and the field $\xi(\mathbf{r})$ is a Lagrange multiplier maintaining incompressibility. The $\left\{\alpha_{i}\right\}$ are the sphere-to -diblock volume ratios, given by

$$
\alpha_{i}=\frac{v_{R_{i}} \rho_{0}}{N}=\frac{4 \pi}{3}\left(\frac{R_{i}}{R_{0}}\right)^{3} \bar{N}^{1 / 2},
$$

where $R_{0}=a N^{1 / 2}$ is the rms end-to-end size of a copolymer, and $\bar{N}=a^{6} \rho_{0}^{2} N$ is the invariant polymerization index. Also, $N$ is the number of segments of volume $\rho_{0}^{-1}$ in a diblock, whereas $a$ is the statistical segment length. The partition functions $Q_{d}$ and $Q_{i}$ of single diblocks or particles, respectively, in appropriate fields have been given in previous work [8], and are

$$
\begin{gathered}
Q_{d}=\int \widetilde{\mathcal{D}} \mathbf{r} \exp \left[-\int_{0}^{f} d s w_{A}(\mathbf{r}(s))-\int_{f}^{1} d s w_{B}(\mathbf{r}(s))\right], \\
Q_{i}=\int d \mathbf{r} \exp \left[-w_{i}(\mathbf{r})\right] .
\end{gathered}
$$

The mean field expression (2) lacks the steric interactions between particles required to properly account for excluded volume. We therefore add to it DFT terms based on the theory of Denton and Ashcroft [19]. The full free energy is then written as 


$$
\begin{aligned}
\frac{N F}{\rho_{0} k_{B} T V}= & -\sum_{i} \frac{\phi_{i}}{\alpha_{i}} \ln \left(\frac{Q_{i} \alpha_{i}}{V \phi_{i}}\right) \\
& -\left(1-\sum_{i} \phi_{i}\right) \ln \left[\frac{Q_{d}}{V\left(1-\sum_{j} \phi_{j}\right)}\right] \\
& +\frac{1}{V} \int d \mathbf{r}\left[\frac{1}{2} \sum_{\mu, \nu} \chi_{\mu \nu} N \varphi_{\mu} \varphi_{\nu}-\sum_{k=A, B} w_{k} \varphi_{k}\right. \\
& -\sum_{i} w_{i} \rho_{i}-\xi\left(1-\sum_{\mu} \varphi_{\mu}\right) \\
& \left.+\sum_{i} \rho_{i} \Psi\left(\bar{\varphi}_{i},\left\{x_{j}\right\}\right)\right]
\end{aligned}
$$

Here $\Psi$ is the nonideal steric term that takes into account the excluded volume interactions between the particles. It is a function of the number (mole) fraction of the various particle species and of the "weighted" densities $\left\{\bar{\varphi}_{i}(\mathbf{r})\right\}$. The number fractions in terms of the overall volume fractions are

$$
x_{i}=\frac{\phi_{i}}{\alpha_{i} \sum_{j} \frac{\phi_{j}}{\alpha_{j}}},
$$

and the weighted densities are given by

$$
\bar{\varphi}_{i}(\mathbf{r})=\sum_{j} \frac{\alpha_{j}}{v_{\left(R_{i}+R_{j}\right)}} \int_{\left|\mathbf{r}^{\prime}\right|<R_{i}+R_{j}} d \mathbf{r}^{\prime} \rho_{j}\left(\mathbf{r}+\mathbf{r}^{\prime}\right) .
$$

Here, $v_{\left(R_{i}+R_{j}\right)}$ denotes the volume of a sphere of radius $R_{i}$ $+R_{j}$. Equation (8) uses the Tarazona form [20] of the weighted density approximation (WDA). Although there exist many more sophisticated WDA choices, we select this form because it is compact, computationally efficient, and gives the correct freezing transition in the case of monodisperse spheres [20]. In choosing the form of the excess free energy per particle $\Psi$, we have taken the expression of Mansoori et al. [21]. This reduces to the Carnahan-Starling equation of state [22] for the case of monodisperse spheres, and so makes this work consistent with our previous results $[7,8]$ and with the approach of Tarazona [20].

The mean field equations describing the possible morphologies are acquired by extremizing the free energy in Eq. (6). These are

$$
\begin{gathered}
w_{i}(\mathbf{r})=\sum_{\mu \neq i} \chi_{i \mu} N \varphi_{\mu}(\mathbf{r})+\xi(\mathbf{r}), \\
\varphi_{i}(\mathbf{r})=\frac{\left(1-\sum_{j} \phi_{j}\right) V}{Q_{d}} \int d s q(\mathbf{r}, s) q^{\dagger}(\mathbf{r}, s),
\end{gathered}
$$

where $i=A, B$, and

$$
\begin{aligned}
& w_{i}(\mathbf{r})= \Psi\left(\bar{\varphi}_{i},\left\{x_{j}\right\}\right)+\frac{\alpha_{i}}{v_{R_{i}}} \int_{\left|\mathbf{r}^{\prime}\right|<R_{i}} d \mathbf{r}^{\prime}\left[\sum_{\mu \neq i} \chi_{\mu i} N \varphi_{\mu}\left(\mathbf{r}+\mathbf{r}^{\prime}\right)\right. \\
&\left.+\xi\left(\mathbf{r}+\mathbf{r}^{\prime}\right)\right]+\sum_{j} \frac{\alpha_{j}}{v_{R_{i}+R_{j}}} \\
& \times \int_{\left|\mathbf{r}^{\prime}\right|<\left(R_{i}+R_{j}\right)} d \mathbf{r}^{\prime} \rho_{j}\left(\mathbf{r}+\mathbf{r}^{\prime}\right) \Psi^{\prime}\left(\bar{\varphi}_{j},\left\{x_{k}\right\}\right) \\
& \rho_{i}(\mathbf{r})=\frac{\phi_{i} V}{\alpha_{i} Q_{i}} \exp \left[-w_{i}(\mathbf{r})\right] \\
& \sum_{\mu} \varphi_{\mu}(\mathbf{r})=1
\end{aligned}
$$

where $i=p_{1}, \ldots, p_{m}$, with

$$
\begin{aligned}
Q_{d} & =\int d \mathbf{r} q(\mathbf{r}, s) q^{\dagger}(\mathbf{r}, s), \\
Q_{i} & =\int d \mathbf{r} \exp \left[-w_{i}(\mathbf{r})\right],
\end{aligned}
$$

and $\Psi^{\prime}\left(\bar{\varphi},\left\{x_{i}\right\}\right)=\partial \Psi\left(\bar{\varphi},\left\{x_{i}\right\}\right) / \partial \bar{\varphi}$. The integral over $s$ in Eq. (10) runs from 0 to $f$ for the $A$ block and from $f$ to 1 for the $B$ block, $f$ being the fraction of $A$ segments per chain. The propagators $q$ and $q^{\dagger}$ are solutions to modified diffusion equations [15]. The real space algorithm for solving the system of equations (9)-(15) has been outlined elsewhere [8]. We now examine some specific systems described by the above formalism.

\section{RESULTS AND DISCUSSION}

In this paper, we are interested in studying binary hard sphere/diblock copolymer mixtures. Therefore we choose $m$ $=2$, and throughout, one group of spheres is taken to have radius $R_{1}=0.2 R_{0}$, and the other group having radius $R_{2}$ $=0.1 R_{0}$. For convenience, we refer to these as "large" and "small" spheres, respectively [23]. To be consistent with previous research $[7,8]$, we take a representative invariant polymerization index of $\bar{N}=1000$, and the diblock segregation as $\chi_{A B} N=20$. Furthermore, to keep the parameter space manageable, let us consider the important case of preferential wetting, where $\chi_{A p_{1}} N=\chi_{A_{p_{2}}} N=\chi_{p_{1} p_{2}} N=0$, and $\chi_{B p_{1}} N$ $=\chi_{B p_{2}} N=\chi_{A B} N$. This implies that both sizes of particles are chemically identical to the $A$ species. For a volume fraction of monodisperse particles of $15 \%$, it was previously found that the system exhibits a lamellar morphology when $f$ $=0.35$ [7]. We select this same value of $f$ for the present bidisperse case, and also choose $\phi_{p_{1}}=0.1$ while $\phi_{p_{2}}=0.05$ [24].

A lamellar morphology is also found for these parameter choices and the density profiles for this system are shown in Fig. 1. As seen in Fig. 1(a), the larger particles (dashed curve) segregate in the center of the shorter $A$ block (solid curve), while the smaller spheres (dotted curve) are expelled to the edge of the $A$ phase, and to a large degree into the $B$ 

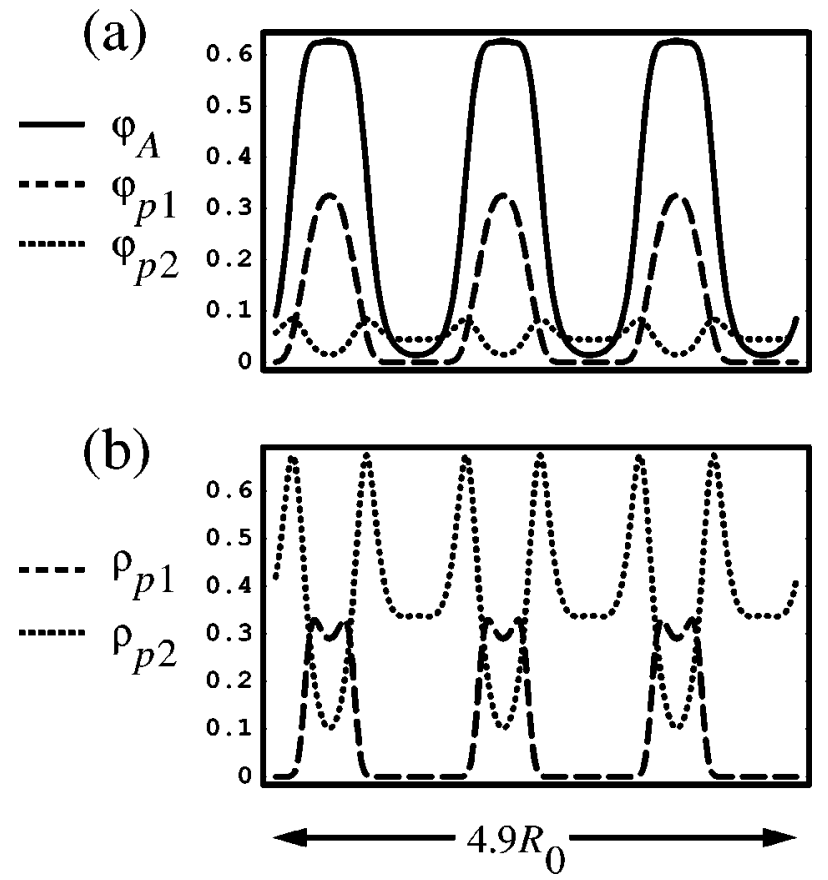

FIG. 1. Dimensionless density profiles of a bidisperse particle and diblock copolymer system with a fraction of $A$ segments per chain of $f=0.35$. (a) The solid curve represents the $A$-block distribution, the dashed curve shows the distribution of the $R_{1}=0.2 R_{0}$ ("large") particles and the dotted curve shows the $R_{2}=0.1 R_{0}$ ("small") particles. The volume fractions are $\phi_{1}=0.1$ and $\phi_{2}$ $=0.05$, respectively. (b) The dashed curve shows the distribution of the centers of the large particles, while the dotted curve shows the small particle center distribution.

domain ( $B$ density profiles are not depicted). Since all the particles (large and small) are of the $A$ chemical species, there is no enthalpic reason for the small particles to coalesce in the $B$ region or in the $A B$ interfacial region. Rather, the particle microphases separate based on the size polydispersity due to the high effective concentration caused by the spheres' spatial confinement within the diblock matrix. This size-based microphase separation is also apparent by examining the distribution of the centers of the particles as shown in Fig. 1(b). Again, one can see a significant number of small particles (dotted curve) that are separated from the region of large particles (dashed curve), where there are very few small particles.

It can be shown that this entropic self-assembly of large and small particles is a result of size polydispersity. Figure 2 shows the density profiles of the system just discussed in the limit that the small particles are set to be the same size as the large particles, that is, they all have radii of $0.2 R_{0}$. In fact, we have monodispersity in this case, but we continue to display the two-particle groups separately for convenience. Figure 3(a) shows the free energy of this system as $R_{2}$ is gradually decreased from $R_{2}=0.2 R_{0}$ to $R_{2}=0.1 R_{0}$, changing the system from monodisperse to our standard bidisperse case. The solid curve indicates the case where the final morphology with $R_{2}=0.1 R_{0}$ reverts to that shown in Fig. 1 . In contrast, the dashed line represents the case where the particles in Fig. 2 are "frozen" in place, so that they cannot demix as
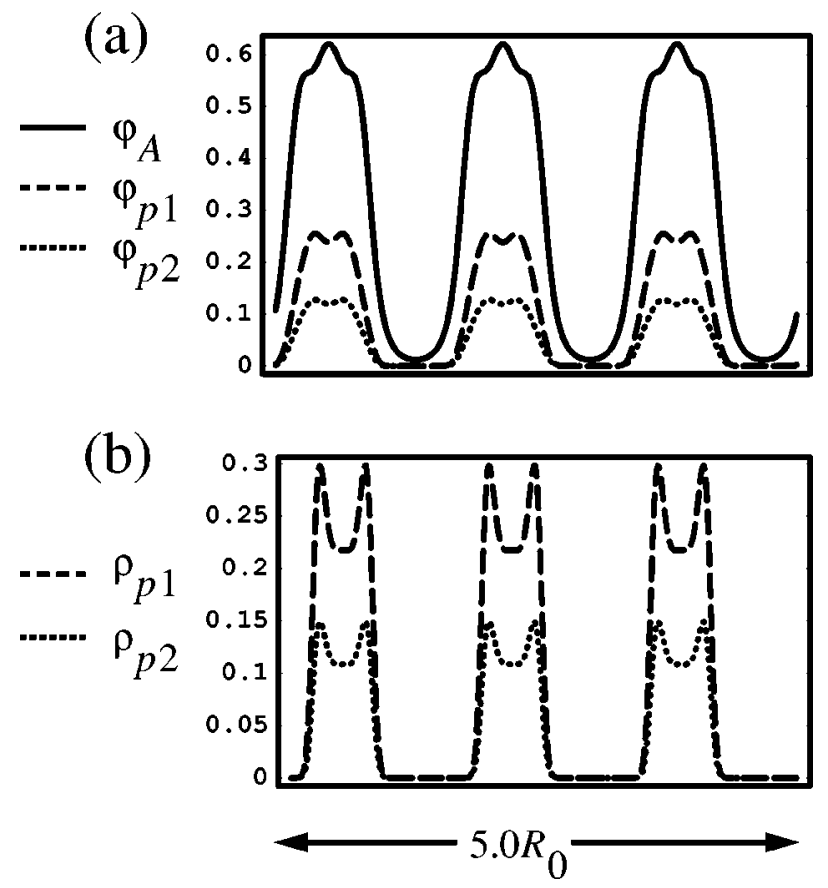

FIG. 2. Same as Fig. 1, except all the particles depicted are of a radius $0.2 R_{0}$. (a) The $A$ block is shown by the solid line with twothirds of the particles shown by the dashed curve, and the remainder by the dotted curve. In this way, this also represents a bidisperse particle morphology where mixing of the particles is enforced with $R_{1}=0.2 R_{0}$ and $\phi_{1}=0.1$, shown by the dashed curve, while $R_{2}$ $=0.1 R_{0}$ with $\phi_{2}=0.05$ is shown by the dotted curve. (b) Distribution of the centers of the particles. Again, this represents either a monodisperse system of large particles or a "frozen" distribution of bidisperse particles.

the particle size difference increases. The final morphology (where $R_{2}=0.1 R_{0}$ ) for this case is forced to remain very much like Fig. 2, but the free energy now increases [see dashed line in Fig. 3(a)].

The cause for this energy penalty of mixing the different sized particles can be traced by decomposing the free energy into components; the main contributions are shown in Figs. 3(b)-3(d). In Fig. 3(b) we see the enthalpic contribution to the free energy of the unfrozen and frozen cases shown by the solid and dashed lines, respectively. As the small particles migrate out of the $A$ phase into the $B$ for the unfrozen case, the enthalpy contribution goes up, as expected. This is more than compensated, however, by two factors: the steric term associated with the small particles, given by

$$
S_{s 2}=\frac{1}{V} \int d \mathbf{r} \rho_{p 2}(\mathbf{r}) \Psi\left(\bar{\varphi}_{p 2},\left\{x_{i}\right\}\right),
$$

which is the $i=2$ term of the last summation in Eq. (6) [plotted in Fig. 3(c)], and the small-particle translational entropy [shown in Fig. 3(d)]. Clearly, it is Eq. (16) that is mostly responsible for the unfavorable free energy of the morphology shown in Fig. 2. The steric term (16) represents the deviation of the particle free energy from the inhomogeneous ideal gas expression due to the particle excluded volume. Specifically, the excess free energy per particle $\Psi$ for 

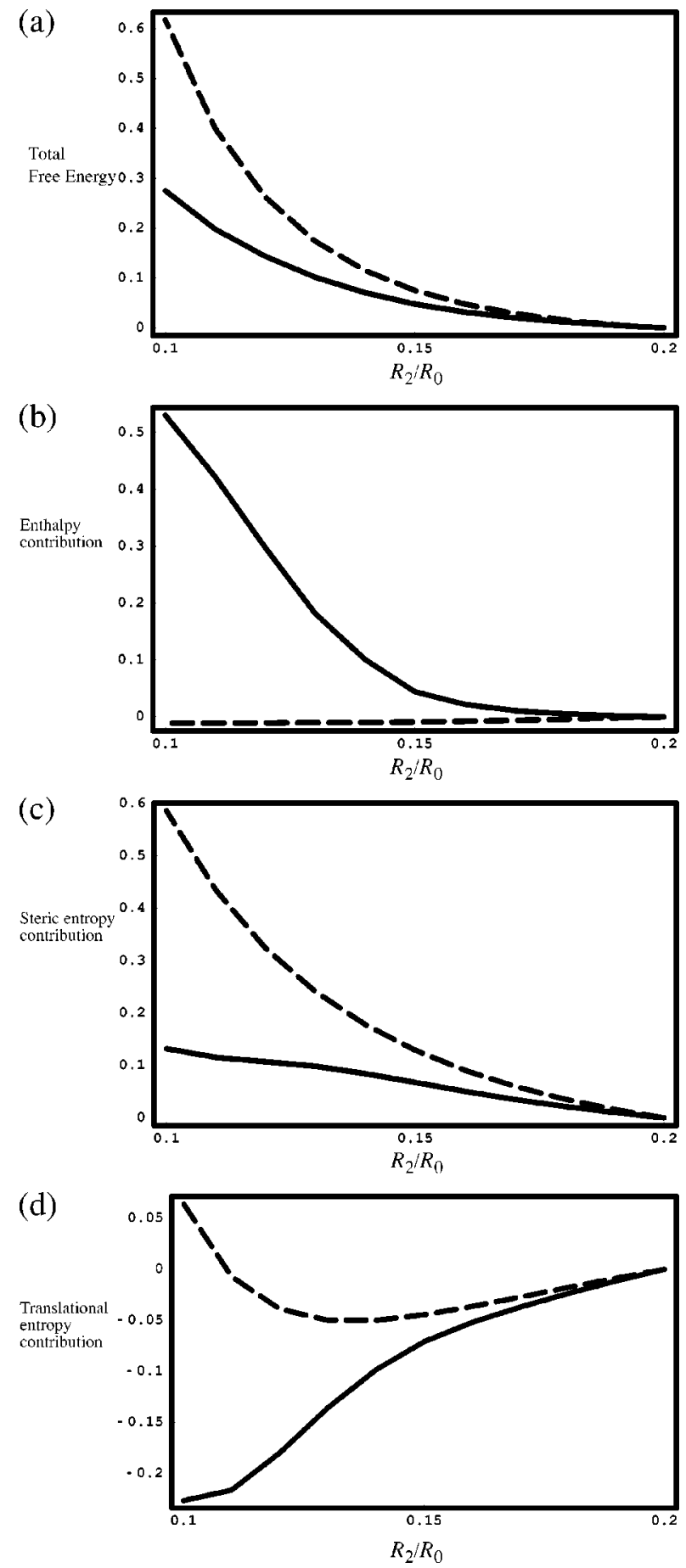

FIG. 3. Free energy versus small particle size for a system of bidisperse particles and diblock copolymer melt. (The free energy is dimensionless.) When $R_{2}=0.2 R_{0}$, the system is monodisperse with a morphology shown in Fig. 2. The dashed line represents the free energy of the system when this morphology is enforced irrespective of the small particle size. The solid line shows the free energy when the morphology is free to adopt the lowest energy configuration. (a) The total free energy of the system. (b) The enthalpic contribution to the free energy. (c) The steric contribution to the free energy associated with the small particles, given by Eq. (16). (d) The translational entropy (ideal entropy) contribution to the free energy of the small particles.
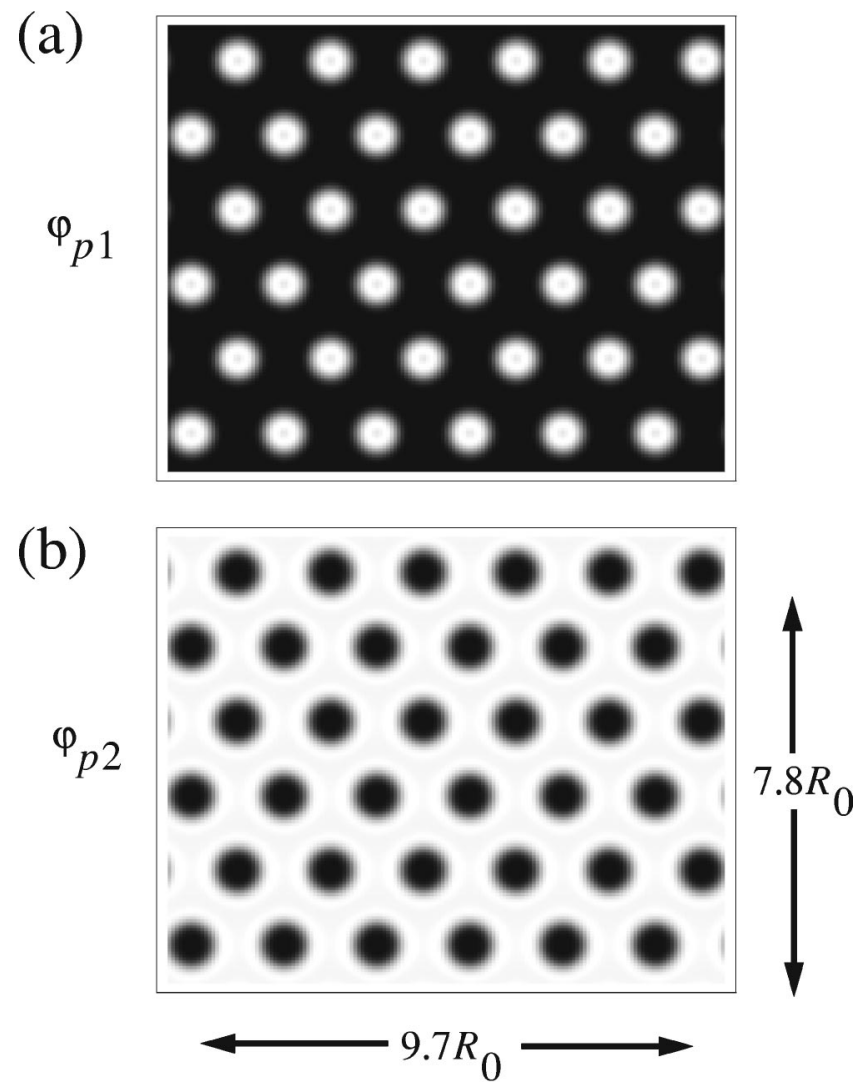

FIG. 4. Dimensionless density plots for a cylindrical phase diblock copolymer and bidisperse spherical particle system with $f$ $=0.2$. Particle sizes and volume fractions are as in Fig. 1. (a) Large particles; white areas indicate a high local volume fraction, whereas dark regions indicate a low volume fraction. (b) Small particles; in both figures, the contrast has been enhanced to clarify the pictures. $A$ and $B$ block regions are not depicted.

the morphology in Fig. 2 is entropically less desirable than for relatively monodisperse local regions shown in Fig. 1.

The polydispersity induced particle microphase separation shown here for the lamellar phase is quite robust for the particular particle radii and particle volume fractions mentioned earlier. Figure 4 shows the density plots for large [Fig. 4(a)] and small [Fig. 4(b)] particles in a cylindrical morphology with $f=0.2$. We can see that the large particles segregate within the cylinders while the small particles exist primarily in the wrong phase. The same phenomenon can be seen in the spherical morphology, as shown in Fig. 5. Figure 5(a) shows the spherical distribution of the $A$-block monomers through an isosurface of $\varphi_{A}(\mathbf{r})=0.21$. Figures 5(b) and 5(c) show the large and small particle distribution cross sections, respectively. In Fig. 5, we have reduced the particle volume fractions to $\phi_{p 1}=0.03$ and $\phi_{p 2}=0.02$, and the $A$-block chain fraction is now $f=0.21$. The cylindrical and spherical phases exhibit the same substructure observed in the lamellar morphology; we hypothesize that this "submorphology" is likely present in all the different phases for this system.

The expulsion of some $A$-like particles from the $A$-rich region to the $B$-rich region can yield a decrease in the "effective" value of $f$ [9], and consequently, could result in transition from one mesophase to another (e.g., from lamellar to 


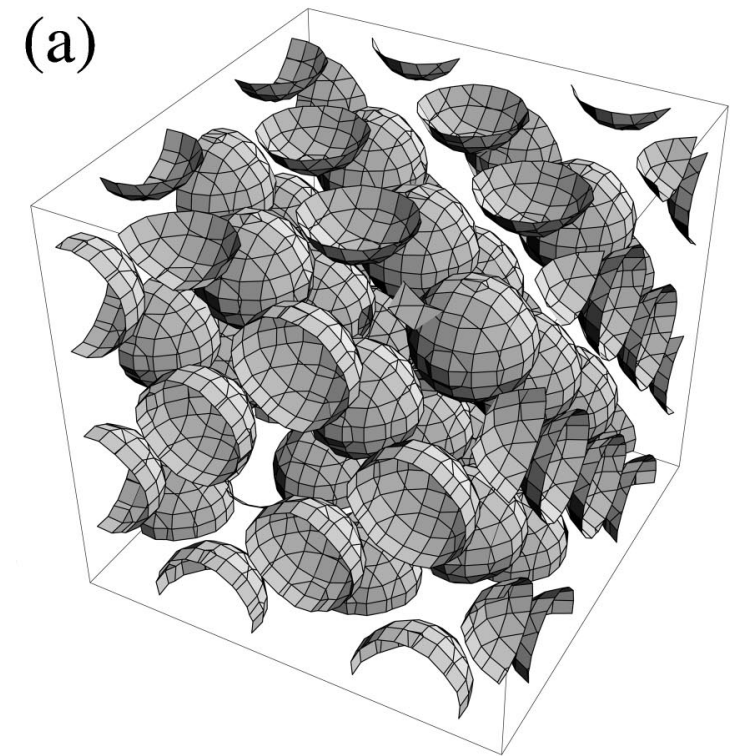

(b)

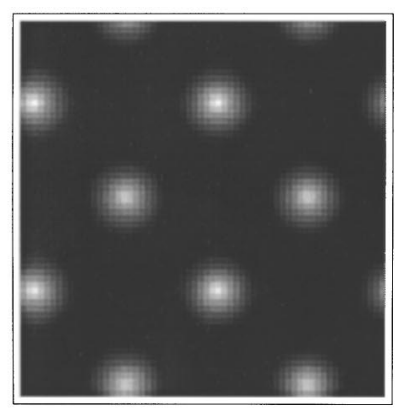

(c)

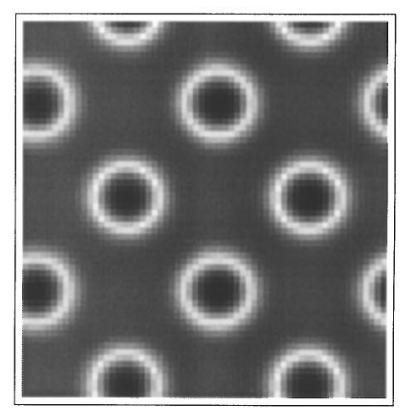

FIG. 5. Spherical morphology. The particle volume fractions are now $\phi_{p 1}=0.03$ and $\phi_{p 2}=0.02$, while the $A$ chain fraction is $f$ $=0.21$. The system dimensions are $7.7 R_{0} \times 7.7 R_{0} \times 7.4 R_{0}$. (a) An $A$-block isosurface at $\varphi_{A}(\mathbf{r})=0.21$, showing the bcc spherical pattern. (b) Cross section showing the distribution of large particles. White areas indicate a high local volume fraction, whereas dark regions indicate a low volume fraction. (c) Cross section showing the small particle distribution. In both cross sections, the contrast has been enhanced to clarify the pictures.

cylindrical). This type of transition is more likely to occur if the system is near an order-order transition (OOT). We have observed such morphological changes as a result of altering the size of monodisperse spherical particles in a diblock matrix [9]. In the present work, however, the volume fraction of particles forced into the $B$ phase as a result of the polydispersity is relatively small, and so an OOT might not be expected to occur. We plan to examine this phenomenon using both SCFT/DFT and strong segregation theory, where we will consider a more significant difference in particle sizes, and higher volume fractions of particles [25].

We now turn our attention to a different binary sphere system, where the particles are all of the same size, but are of different chemical species. It has been predicted [8] that a small volume fraction of particles attracted to the minority phase of a diblock copolymer can form nanowires in a hexagonal pattern. Similarly, particles attracted to the majority (a)

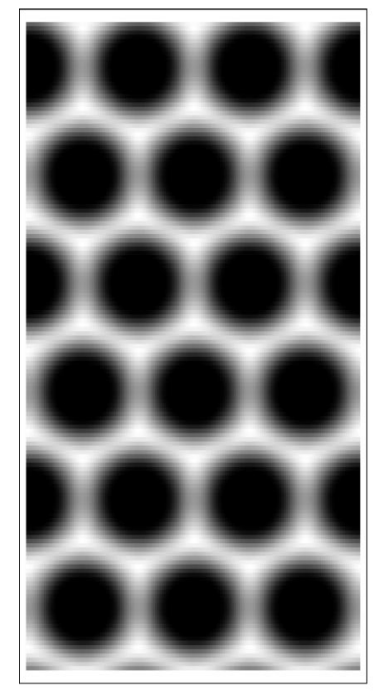

(b)

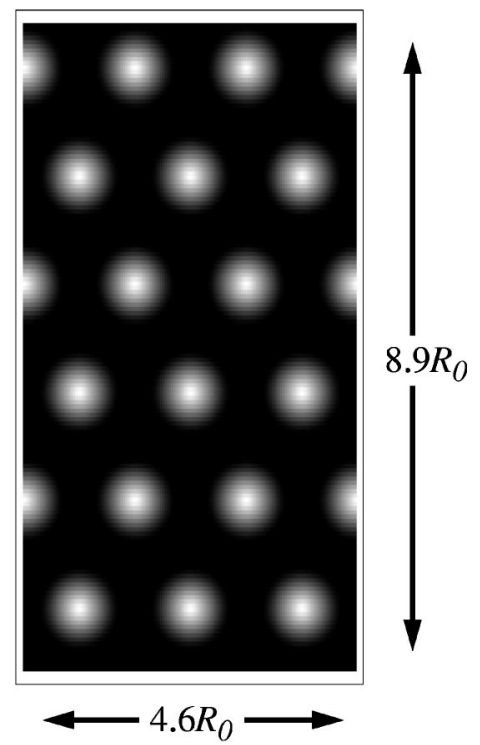

FIG. 6. Dimensionless density plots for a threaded cylindrical phase of an $A B$ diblock copolymer and $A B$ particle mixture. All particles are of the same size with $R_{1}=R_{2}=0.3 R_{0}$, and have equal volume fractions with $\phi_{A}=\phi_{B}=0.075$. The $A$-block chain fraction is $f=0.7$. (a) $A$-type particles forming a matrix structure. White areas indicate a high local volume fraction, whereas dark regions indicate a low volume fraction. (b) $B$-type particles forming nanowires that thread the hexagonal matrix. In both figures, the contrast has been enhanced to clarify the pictures. $A$ and $B$ block regions are not depicted.

phase can spread through the hexagonal matrix to form an inverted structure. One would therefore expect that if twoparticle types (each with an affinity for opposite blocks of a copolymer) were mixed with an asymmetric diblock, the system could self-assemble into a particle-filled hexagonal matrix that is threaded by nanowires.

We can test this expectation using the current theory. We again take $m=2, \bar{N}=1000, \chi_{A B} N=20$, and now we set $\chi_{A p_{1}} N=\chi_{B p_{2}} N=0$ while $\chi_{A p_{2}} N=\chi_{B p_{1}} N=\chi_{p_{1} p_{2}} N=\chi_{A B} N$. This implies that the $p_{1}$ group of particles are coated in $A$ monomers, while the $p_{2}$ group is coated in $B$. We find that we can realize such a threaded structure if we choose particle sizes $R_{1}=R_{2}=0.3 R_{0}$ and an $A$ block chain fraction of $f$ $=0.7$. We keep the overall volume fraction of particles at $15 \%$, letting half of it be $A$ particles and half be $B$ particles. The resulting morphology is shown in Fig. 6. Figure 6(a) shows the $A$-type particles, whereas Fig. 6(b) shows the $B$-type particles.

\section{CONCLUSIONS}

We described a general theory, which is based on an SCFT/DFT hybrid methodology, for mixtures of polydisperse, hard spheres, and diblock copolymers. We used this theory to examine two different binary sphere/diblock mixtures and found that the introduction of physically or chemically distinct particles can be exploited to control the equi- 
librium morphology of the system.

In the first system, we chose chemically identical spherical particles of two different sizes, where both the large and small particles have a preferential attraction to one of the blocks of the copolymer. We found that in the equilibrium morphology, the particles undergo a kind of "demixing," where the large particles embed themselves in the preferred, compatible phase, while the smaller particles spread out in interfacial regions and in the incompatible phase of the diblock. This is shown in Fig. 1. We suggest that this effect will be found in other more complex morphologies, not just the ones shown here.

We also studied a second system where we chose the particles to be the same size, but of two different chemical species. Here, half the particles favor $A$-rich regions and the other half favor $B$-rich regions. We observed a spatially ordered nanocomposite, where one species of particles forms a hexagonal matrix, whereas the other forms nanowires that weave through the first. This is shown in Fig. 6.
Both systems are examples of using binary spheres in a diblock melt to control the self-assembly and structure of organic/inorganic hybrid materials. The first one shows an entropic separation of particles in the diblock matrix, whereas the second one shows an enthalpic separation. The SCFT/DFT approach presented here can readily be extended to other block copolymer architectures, and thus, the model can provide guidelines for controlling structure formation in a variety of block copolymer/spherical colloid mixtures.

\section{ACKNOWLEDGMENTS}

The authors acknowledge helpful communication with V. V. Ginzburg. This work was supported by the U.S. Department of Energy, NSF, and the Army Research Office. Extensive use was made of the Center for Molecular and Materials Simulations computing facilities at the University of Pittsburgh.
[1] A.C. Balazs, Curr. Opin. Colloid Interface Sci. 4, 443 (2000).

[2] D.M. Dabbs and I.A. Aksay, Annu. Rev. Phys. Chem. 51, 601 (2000), and references therein.

[3] W.A. Lopes and H.M. Jaeger, Nature (London) 414, 735 (2001), and references therein.

[4] P.F.W. Simon, R. Ulrich, H.W. Spiess, and U. Wiesner, Chem. Mater. 13, 3464 (2001), and references therein.

[5] A.C. Edrington, A.M. Urbas, P. DeRege, C.X. Chen, T.M. Swager, N. Hadjichristidis, M. Xenidou, L.J. Fetters, J.D. Joannopoulos, Y. Fink, and E.L. Thomas, Adv. Mater. 13, 421 (2001), and references therein.

[6] J. Huh, V.V. Ginzburg, and A.C. Balazs, Macromolecules 33, 8085 (2000).

[7] R.B. Thompson, V.V. Ginzburg, M.W. Matsen, and A.C. Balazs, Science 292, 2469 (2001).

[8] R.B. Thompson, V.V. Ginzburg, M.W. Matsen, and A.C. Balazs, Macromolecules 35, 1060 (2002).

[9] J.Y. Lee, R.B. Thompson, D. Jasnow, and A.C. Balazs, Macromolecules 35, 4855 (2002).

[10] M. Adams, Z. Dogic, S.L. Keller, and S. Fraden, Nature (London) 393, 349 (1998).

[11] A.G. Yodh, K.-H. Lin, J.C. Crocker, A.D. Dinsmore, R. Verma, and P.D. Kaplan, Philos. Trans. R. Soc. London, Ser. A 359, 921 (2001), and references therein.

[12] A.D. Dinsmore, A.G. Yodh, and D.J. Pine, Nature (London) 383, 239 (1996).
[13] M. Dijkstra, R. van Roij, and R. Evans, Phys. Rev. E 59, 5744 (1999).

[14] N.A. Kotov, MRS Bull. 26, 992 (2001).

[15] M.W. Matsen, J. Phys.: Condens. Matter 14, R21 (2002).

[16] G.H. Fredrickson, V. Ganesan, and F. Drolet, Macromolecules 35, 16 (2002).

[17] R. Evans, in Fundamentals of Inhomogeneous Fluids, edited by D. Henderson (Dekker, New York, 1992), pp. 85-175.

[18] The form of Eqs. (2) and (6) assumes the various particle types are distinguishable.

[19] A.R. Denton and N.W. Ashcroft, Phys. Rev. A 42, 7312 (1990).

[20] P. Tarazona, Mol. Phys. 52, 81 (1984).

[21] G.A. Mansoori, N.F. Carnahan, K.E. Starling, and T.W. Leland, J. Chem. Phys. 54, 1523 (1971).

[22] N.F. Carnahan and K.E. Starling, J. Chem. Phys. 51, 635 (1969).

[23] It should be noted that in our previous work [7,8], we took "large" particles to have radii of $0.3 R_{0}$, while "small" particles had radii of $0.2 R_{0}$. The reader should take care to notice that we are now using two different sizes of particles when speaking of large or small spheres.

[24] We chose the values of the volume fractions such that they are low enough to avoid macrophase separation of the particles, but high enough to reveal interesting effects. Also, too high volume fractions of particles can cause numerical instability in the algorithm.

[25] J. Y. Lee, R. Thompson, D. Jasnow, and A. C. Balazs, J. Chem. Soc., Faraday Discussions (to be published). 